\title{
Regulation of Hypoxia-Induced Cell Death and Application of Hypoxic Preconditioning to Stem Cell Transplantation
}

\begin{abstract}
Keywords: Apoptosis; Hypoxia; Stem cell; Transplantation; Stem cell therapy

Abstract

Hypoxia is a commonly encountered feature of the cellular microenvironment in a number of processes in which programmed cell death (apoptosis) affects disease progression. These diseases include myocardial infarction, stroke, and ischemic acute kidney injury. Bone marrow-derived mesenchymal stem cells (MSCs) are multipotent adult stem cells that are able to differentiate into endothelial cells, vascular smooth muscle cells, or cardiac-like myocytes when transplanted into the ischemic heart. Both animal and clinical studies have substantiated that MSC transplantation can enhance cardiac function through possible angiogenesis and myogenesis after myocardial infarction. However, some studies have failed to monitor the therapeutic effects of MSC transplantation; therefore, there is a need for supplementary research on the use of MSCs and the improvement of transplantation techniques after MI. An important problem in stem cell therapy for ischemic heart diseases is the low survival of transplanted cells in the ischemic and infarcted sites. Most transplanted cells die within 4 days after transplantation into the ischemic heart. Endogenous and environmental factors, such as hypoxia and inflammatory response, may contribute to cell death. Therefore, enhancing implanted cell survival after transplantation is vital for improving the effect and efficiency of stem cell therapy. In this review, we investigate whether hypoxia is responsible for activating apoptosis signaling in transplanted stem cells, and could be a potential target for enhancing the therapeutic effect of stem cells in treating ischemic heart diseases.
\end{abstract}

\section{Abbreviations}

NOS: Nitric Oxide Synthase; PDK: Pyruvate Dehydrogenase Kinase; polyp: Polyphosphate; PTP: Permeability Transition Pore; ROS: Reactive Oxygen Species; SDF-1: Stromal-Derived Factor-1; UCP: Uncoupling Protein; VEGF: Vascular Endothelial Growth Factor

\section{Introduction}

Hypoxia occurs when oxygen demand exceeds supply [1]. Hypoxia causes ATP levels to drop and inhibits maintenance of cellular functions; if the condition lasts for sufficient time, cells die. Severe hypoxia results in a high mutation rate, causing point mutations that may be explained by reduced DNA mismatch repair activity resulting from hypoxia-induced decreases in NLH1 and PMS2 concentrations [2]. In addition, hypoxia induces genetic instability by the induction of fragile sites causing gene amplification [3-5]. Therefore, during severe hypoxia or anoxia, the cell initiates a cascade of events that leads to apoptotic cell death, thereby preventing the accumulation of cells with hypoxia-induced mutations [6]. A classic example of an acute hypoxic event is stroke. Although a stroke lesion can lead to both types of cell death (i.e., necrosis and apoptosis), it is typically characterized by a core of necrosis $[7,8]$, which is a passive,

\section{Journal of}

\section{Transplantation \&} Stem Cell Biology

\author{
Yong-Seok Han ${ }^{1 \#, ~ J u n ~ H e e ~ L e e ~}{ }^{2 \#}$ and Sang \\ Hun Lee ${ }^{1 *}$ \\ ${ }^{\prime}$ Medical Science Research Institute Soonchunhyang University, \\ Seoul Hospital, Seoul 336-745, Korea, and Department of Medical \\ Bioscience, Soonchunhyang University, Asan 336-745, Korea \\ ${ }^{2}$ Laboratory for Vascular Medicine \& Stem Cell Biology, Medical \\ Research Institute, Department of Physiology, School of Medicine, \\ Pusan National University, Yangsan 626-870, Korea \\ "These author's contributed equally \\ Address for Correspondence \\ Sang Hun Lee, Medical Science Research Institute Soonchunhyang \\ University, Seoul Hospital, Seoul 336-745, Korea, Tel: 02-709-9029; Fax: \\ 82-2-792-5812; Email: jhlee0407@sch.ac.kr \\ Submission: 03 September 2014 \\ Accepted: 13 September 2014 \\ Published: 18 September 2014 \\ Reviewed \& Approved by: Dr. Pranela Rameshwar, Division of \\ Hematology and Oncology, New Jersey Medical School, USA
}

uncontrolled type of cell death. Similar cascades are thought to occur during myocardial infarction, which is also characterized by a core of necrosis and a border zone in which cells undergo apoptosis $[9,10]$.

Although diverse treatment for cardiac diseases has been developed, heart failure remains a critical disease [11] because regenerative ability after myocardial infarction (MI) is limited [1214]. Recently, therapeutic approaches have used genes, growth factors, or cells to enhance myocardial substitutes [15]. Given recent progress in restoring the ischemic heart using mesenchymal stem cells (MSCs), understanding the tissue microenvironment will help optimize the therapeutic potential of these cells [16]. However, MSCs transplanted into an infarcted site of the ischemic heart encounter severe conditions such as calcium excess, acidosis, and reactive oxygen species (ROS), which induce apoptotic signaling and inhibit survival [17-20]. Ultimately, ROS production in the injured heart induces apoptosis in both implanted cells and cardiomyocytes [2123].

Apoptosis regulatory genes have been the subject of much research. Apoptosis is regulated by both activators and repressors. The balance of these molecules determines whether apoptosis is initiated. Recent research has suggested that some oncogenes and tumor suppressor genes control programmed cell death. Bcl-2, p53, and $c-M y c$ are known to be apoptosis regulatory genes [24-26]. In addition, $B c l-x L, B c l-W$, and $M c l-1$ are well-established apoptosis inhibitors. In contrast, Bad, Bid, Bim, Bmf, Bik, Hrk, Noxa, and Puma are known to induce apoptosis [27].

In this review, we investigate whether hypoxia-induced apoptosis is a strong inducer of cell death of implanted MSCs in the ischemic heart, and whether it makes a promising target for enhancing the therapeutic effect of MSCs in clinical research.

\section{Inducing Apoptosis under Hypoxia}

Hypoxia induces necrosis and apoptosis [28,29]. Hypoxiainduced apoptosis is related to increased mitochondrial permeability and release of cytochrome $c$ into the cytoplasm, is caspase-9 
dependent but caspase-8 independent, and mainly occurs by way of the intrinsic pathway [30]. However, many cell types endure long periods of hypoxic stress, often requiring additional stressors such as serum deprivation or acidosis for cell death to occur [31-33]. The ability to withstand apoptosis in settings of hypoxic stress is the result of a well-developed cellular response, a phenomenon that is important in many disease processes. The ability to adjust to varying levels of oxygen in the cellular environment is generally attributable to hypoxia-inducible factor-1 (HIF-1). Hypoxia also affects other transcription factors, such as HIF-2, nuclear factor kappaB (NF- $\kappa$ B), and $\mathrm{p} 53$, which play significant roles in the regulation of the apoptotic pathway. In many complex disease processes, the change in hypoxic phenotype has a larger effect on cell survival than the direct induction of apoptosis.

\section{Apoptotic Pathways}

\section{Extrinsic pathway}

Apoptosis is triggered by two signaling pathways. The extrinsic pathway is activated by binding of pro-apoptotic ligands to death receptors on the cell surface. Receptors such as CD95 or tumor necrotic factor receptor 1 (TNF-R1) induce formation of death-inducing signaling complexes (DISCs) comprised of death receptors, TNF receptor-associated death domain (TRADD)-containing proteins, Fas-associated death domain (FADD) adaptor proteins, and initiator caspases. Activation of the death receptor complex following ligand binding promotes interactions between pro-caspase- 8 molecules within the DISC, which subsequently activatecaspase- 3 and induce apoptosis [34-36]. TNF-R1 signaling also promotes cell survival via NF- $\kappa B$ activation, which is stimulated by the recruitment of the TNF receptor associated factor 2 (TRAF2) to ligand-bound receptors. Interestingly, TRADD may serve as a platform for the assembly of TRAF2 or FADD, which determine the subsequent activation of either the NF- $\mathrm{KB}$-induced survival pathway or the caspase-dependent proapoptotic pathway, respectively. In contrast, tumor necrotic factor receptor 1 (TNF-R2) does not include a TRADD motif; thus, TRAF2 can directly interact with NF- $\mathrm{B} B[37,38]$. TNF-R2 also enhances cell survival by stimulating mitogen-activated protein kinases (MAPK).

\section{Intrinsic pathway}

In contrast to the extrinsic pathway, the intrinsic apoptotic pathway involves non-receptor-mediated intracellular signals. These signals are activated by cell stresses such as cell shrinkage, DNA fragmentation, and cytokine deficiency. The intrinsic pathway can also be activated in response to toxins such as chemotherapeutic agents. Intrinsic apoptosis is controlled by the Bcl-2 family of proteins, which are located on the mitochondrial membrane [39]. Members of the Bcl-2 family are classified according to their anti- or pro-apoptotic function. The anti-apoptotic members of this family include Bcl-2, Bcl-xL, Bcl-W, and Mcl-1. In contrast, Bax, Bak, and Bok are pro-apoptotic and Bid, Noxa, Bad, Bim, Bik, Bmf, Hrk, and Puma can induce the expression of apoptotic members of the family. Under normal conditions, the mitochondrial membrane is polarized such that cytochrome $\mathrm{c}$ and ROS are confined within the mitochondrial walls. Pro-apoptotic proteins of the Bcl-2 family stimulate the mitochondrial permeability transport pore and induce cytochrome c release. Once cytochrome c is in the cytoplasm, it binds apoptotic protease activating factor 1 and procaspase- 9 to form an "apoptosome". In the presence of ATP, this complex induces proteolytic cleavage of procaspase- 3 to activated caspase-3. Bax is a well-known pro-apoptotic protein. During apoptosis, Mcl-1 levels are significantly decreased via proteasome degradation. Decreasing levels of Mcl-1 release Bax from the Bak hetero-complex [40,41]. Activated Bax and Bak act as ion channels and connector proteins, allowing for the intermediate release of cytochrome c. In contrast, the anti-apoptotic protein Bcl-2 can inhibit Bax or Bak by enhancing the stability of the mitochondrial membrane or by reducing the transport of pro-apoptotic proteins. Bad and Bid inhibit anti-apoptotic Bcl-2 protein activity and induce pro-apoptotic protein function. Figure 1 shows a schematic of both the extrinsic and intrinsic pathways.

\section{Transcription Factors}

\section{Hypoxia-inducible factor}

Hypoxia-inducible factor (HIF) is a transcription factor that allows cells to adapt to low-oxygen conditions. Hypoxic adaptation is mostly mediated through HIF-1 $\alpha$, which is degraded by prolylhydroxylase (PHD) under normoxic conditions [41]. At hypoxic levels, however, PHD enzymes are inactivated and HIF-1a proteins are free to bind to HIF-1a/aryl hydrocarbon nuclear translocator (ARNT) and p300. p300 then allows the binding of HIF with the promoter region of target genes in the nucleus $[42,43]$. This process allows for enhanced angiogenesis, hematopoiesis, and improved cell survival by enhancing intracellular ATP and oxygen levels and reducing toxic ROS production [41]. In addition, HIF-1 $\alpha$ induces cell cycle arrest in order to reduce the cell's workload [44]. Although HIF-2a is similar to HIF-1 1 , it differs in many important aspects. First, HIF-2 is not ubiquitously expressed and interacts with individual cofactors. For example, HIF-2 does not regulate genes involved in glycolysis, but is the main regulator of erythropoietin in the adult kidney. Moreover, HIF-2 increases cell proliferation via c-Myc $[45,46]$.

\section{NF-kB}

Along with its role in mediating the response of the immune system to infection, NF- $\kappa \mathrm{B}$ regulates the expression of cytokines,

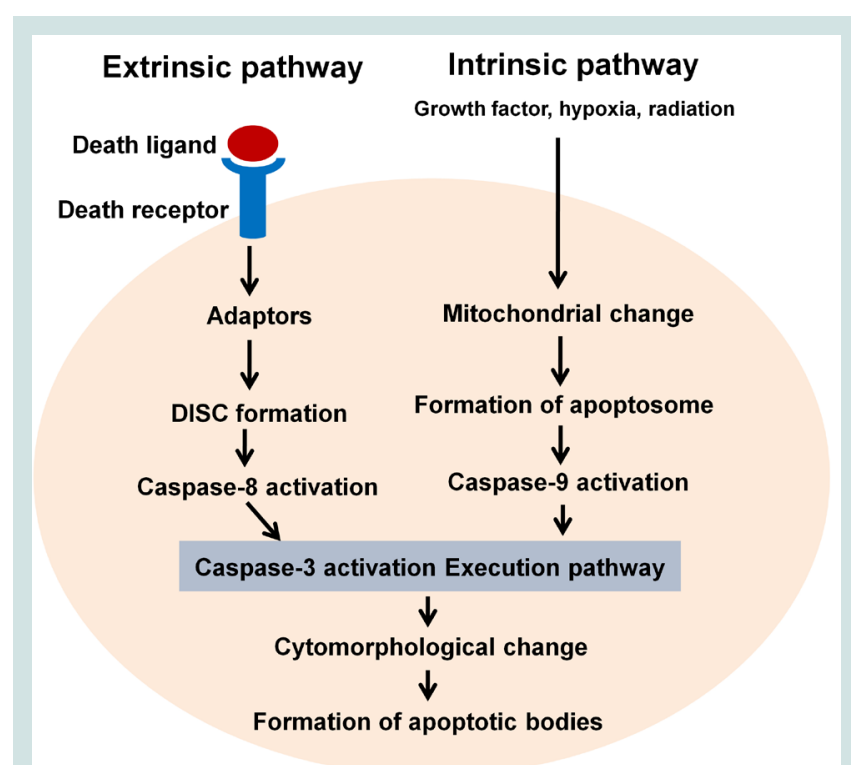

Figure 1: Extrinsic and intrinsic apoptosis pathways. A schematic view of the two main apoptotic pathways: the intrinsic pathway and the extrinsic pathway. 
growth factors, and anti-apoptotic factors [47]. When NF-kB is inactive, its subunits are segregated in the cytoplasm by the inhibitor

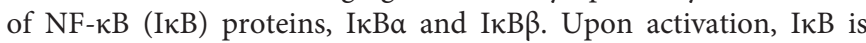
phosphorylated, and its degradation leads to release of bound NF$\mathrm{\kappa B}$ dimers. The free dimers translocate to the nucleus and bind to target promoter or enhancer sites [47]. NF- $\mathrm{kBs}$ are activated via both canonical and noncanonical pathways; however, hypoxia leads only to NF- $\mathrm{kB}$ activation via the canonical pathway [48]. More specifically, low levels of oxygen stimulate IкB kinase (IKK), which leads to the phosphorylation and degradation of I $\mathrm{B}$, and then to NF- $\kappa \mathrm{B}$ activation and knockdown of PHD enzymes [49].

\section{p53}

HIF-1 $\alpha$ is activated under hypoxic conditions, which may have functional significance for the role of the tumor suppressor p53. p53 is involved in the regulation of the cell cycle, DNA repair, and apoptosis. This molecule is induced by pro-apoptotic proteins such as Puma and Noxa. Accumulation of p53 under hypoxic conditions is probably not induced by DNA damage $[1,50]$. Under hypoxic conditions, p53 interacts with transcriptional corepressors such as $\mathrm{mSin} 3 \mathrm{~A} /$ histone deacetylases, inducing apoptosis via transrepression of $\alpha, \beta$-tubulin [51].

\section{Survival pathways involved in hypoxic preconditioning of stem cells}

Because metabolic dysfunction and glutamate excitotoxicity occur in ischemic brain injury, mass apoptosis is induced in always with extra injury from increased ROS, inflammatory responses, activation of apoptotic and other pathological processes. Preconditioning treatments for stem cells have been shown to improve resistance to these insults by increasing anti-apoptotic signals [52,53]. Many survival and defensive molecules, including HIF-1a [54], trophic/growth factors [55], Akt [56,57], extracellular signal-regulated kinase (ERK) [57], glycogen synthase kinase-3 $\beta$ (GSK-3 $\beta$ ), matrix metalloproteinase-2 (MMP-2) [19], survivin [58], and $\mathrm{Bcl}-2$ are engaged in reaction to preconditioning stimuli. Selective up-regulation of these molecules binding to improving defensive signaling is adequately controlled in both preconditioned stem cells and the cells nearby to injury sites [59].

\section{Central roles of HIF-1 in hypoxic preconditioning}

In stem cells, HIF-1 also plays a role in preconditioning and provides an advantage in transplantation therapy (Figure 2). HIF1 induces the cysteine glutamate interchange system of NSCs by increasing expression of the light-chain subunit $\mathrm{xCT}$ [60], which is a limit stage for brain antioxidant glutathione (GSH) production [61]. HIF-1 overexpression in MSCs leads to up-regulation of genes that contribute to cell adhesion, migration, and paracrine effect. Transplantation of these cells into the myocardium of rats after induction of MI improves revival of cardiac functions and angiogenesis $[58,62]$. Collectively, these results suggest that HIF-1 is a significant mediator of stem cell preconditioning (Figure 2).

\section{Hypoxic Preconditioning Provides Therapeutic Benefits in Stem Cell Therapies Enhanced Cell Survival In Vitro and After Transplantation}

The survival rate of implanted cells is a major issue once cells are transplanted into the ischemic heart or brain. The preconditioning factors mentioned here usually confer superior survival of stem cells

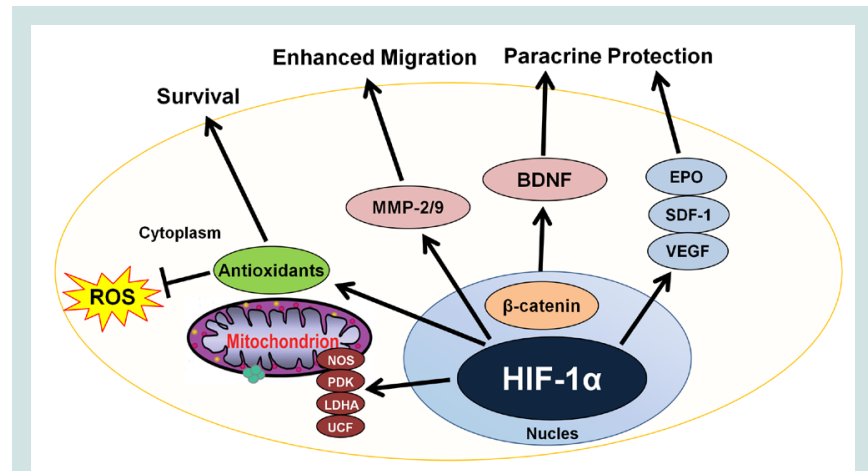

Figure 2: Central roles of HIF-1 signaling pathways in stem cells. Hypoxic preconditioning insults increase HIF-1 expression. HIF-1 then regulates antioxidants, survival signals, and many other genes related to cell adhesion, polarization, migration, and paracrine protection.

and progenitors in vitro or after transplantation. Preconditioning using lethal hypoxia and EPO significantly increased the capacity of treated cells to withstand apoptotic and other stresses in vitro, as well as in the harsh environment of ischemic infarct sites [17,63,64]. MSCs and embryonic stem cell-derived neural progenitor cells (ESNPCs) show improved survival following lethal exposure to hypoxic conditions $\left(1 \% \mathrm{O}_{2}\right)$, as well as a $40-50 \%$ decrease in apoptosis and caspase activation. Hypoxic preconditioning increased the secretion of EPO and up-regulated the expression of Bcl-2, HIF-1, EPO receptor (EPOR), neurofilament (NF), and synaptophysin in ES-NPCs. The defensive effect was decreased by blocking EPOR, and pretreatment of ES-NPCs with recombinant human EPO mimicked the effects of hypoxic preconditioning. Three days after transplantation into the ischemic rat brain, a $30-40 \%$ lower apoptosis was observed in hypoxic preconditioned ES-NPCs as compared to normoxic cells. These surviving ES-NPCs also showed extensive neuronal differentiation in the ischemic brain and improved revival of sensory motor function [64]. A similar survival effect of hypoxia pretreatment has been reported in human ES-NPCs [65]. Previous studies have shown that ES-NPCs have the capacity for peripheral nerve injury recovery [66]. Therefore, improved stem cell survival by preconditioning shows promise for application in cell therapies for tissue repair [67].

\section{Increased regenerative potential of hypoxic-preconditioned} stem and progenitor cells

Preconditioned stem cells show several improved regenerative abilities, including improved migration and homing to lesion sites. In bone marrow-derived hemangioblasts, many chemokine and angiogenic genes were up-regulated after hypoxic induction, which accelerated their differentiation toward the endothelial lineage [68]. Hypoxia also improves the differentiation of EPC-like attaching cells, which promote neovascularization [69]. Their function is dependent on secretion of VEGF and increased VEGF2R expression in reaction to hypoxia [70]. Hypoxic-preconditioned MSCs show increased expression of Wnt4, and induced neovascularization was observed in a murine model of hindlimb ischemia [71]. Another important signaling molecule, sonic hedgehog ( $\mathrm{SHH})$, may be involved in the EPC-mediated angiogenesis and neovascularization induced by VEGF, SDF-1, and angiopoietin-1 [72]. Hypoxic preconditioning of MSCs showed benefits for transplantation therapy for bone regeneration by restoring osteogenic differentiation [73]. 
Citation: Han YS, Lee JH, Lee SH. Regulation of Hypoxia-Induced Cell Death and Application of Hypoxic Preconditioning to Stem Cell Transplantation. J Transplant Stem Cel Biol. 2014;2(1): 5

\section{Conclusion}

Oxygen levels are important in the general physiology of the cell and in instances where cell physiology contributes to disease progression. A better understanding of the transcriptional response to hypoxia and the particular mechanisms underlying hypoxic apoptosis will determine our success in developing therapies that focus on controlling apoptosis induced by disease conditions.

\section{References}

1. Sendoel A, Hengartner MO (2014) Apoptotic cell death under hypoxia. Physiology 29: 168-176.

2. Perou CM, Sorlie T, Eisen MB, van de Rijn M, Jeffrey SS, et al. (2000) Molecular portraits of human breast tumours. Nature 406: 747-752.

3. Rice GC, Hoy C, Schimke RT (1986) Transient hypoxia enhances the frequency of dihydrofolate reductase gene amplification in Chinese hamster ovary cells. Proc Natl Acad Sci U S A 83: 5978-5982.

4. Young SD, Marshall RS, Hill RP (1988) Hypoxia induces DNA overreplication and enhances metastatic potential of murine tumor cells. Proc Natl Acad Sci U S A 85: 9533-9537.

5. Coquelle A, Toledo F, Stern S, Bieth A, Debatisse M (1998) A new role for hypoxia in tumor progression: induction of fragile site triggering genomic rearrangements and formation of complex DMs and HSRs. Mol Cell 2: 259265

6. Reynolds TY, Rockwell S, Glazer PM (1996) Genetic instability induced by the tumor microenvironment. Cancer Res 56: 5754-5757.

7. Lipton P (1999) Ischemic cell death in brain neurons. Physiol Rev 79: 1431 1568.

8. Yuan J (2009) Neuroprotective strategies targeting apoptotic and necrotic cell death for stroke. Apoptosis 14: 469-477.

9. Burke AP, Virmani R (2007) Pathophysiology of acute myocardial infarction. Med Clin North Am 91: 553-572.

10. Krijnen PA, Nijmeijer R, Meijer CJ, Visser CA, Hack CE, et al. (2002) Apoptosis in myocardial ischaemia and infarction. J Clin Pathol 55: 801-811.

11. Fairbanks SL, Brambrink AM (2010) Preconditioning and postconditioning for neuroprotection: the most recent evidence. Best Pract Res Clin Anaesthesio 24: $521-534$

12. Stubbs SL, Hsiao ST, Peshavariya HM, Lim SY, Dusting GJ, et al. (2012) Hypoxic preconditioning enhances survival of human adipose-derived stem cells and conditions endothelial cells in vitro. Stem Cells Dev 21: 1887-1896.

13. Das R, Jahr H, van Osch GJ, Farrell E (2010) The role of hypoxia in bone marrow-derived mesenchymal stem cells: considerations for regenerative medicine approaches. Tissue Eng Part B Rev 16: 159-168.

14. Tilkorn DJ, Davies EM, Keramidaris E, Dingle AM, Gerrand YW, et al. (2012) The in vitro preconditioning of myoblasts to enhance subsequent survival in an in vivo tissue engineering chamber model. Biomaterials 33: 3868-3879.

15. Peterson KM, Aly A, Lerman A, Lerman LO, Rodriguez-Porcel M (2011) Improved survival of mesenchymal stromal cell after hypoxia preconditioning: role of oxidative stress. Life Sci 88: 65-73.

16. Perez-Pinzon MA (2007) Mechanisms of neuroprotection during ischemic preconditioning: lessons from anoxic tolerance. Comp Biochem Physiol A Mol Integr Physiol 147: 291-299.

17. Hu X, Yu SP, Fraser JL, Lu Z, Ogle ME, et al. (2008) Transplantation of hypoxia-preconditioned mesenchymal stem cells improves infarcted heart function via enhanced survival of implanted cells and angiogenesis. J Thorac Cardiovasc Surg 135: 799-808.

18. Aly A, Peterson K, Lerman A, Lerman L, Rodriguez-Porcel M (2011) Role of oxidative stress in hypoxia preconditioning of cells transplanted to the myocardium: a molecular imaging study. J Cardiovasc Surg 52: 579-585.

19. Li JH, Zhang N, Wang JA (2008) Improved anti-apoptotic and anti-remodeling potency of bone marrow mesenchymal stem cells by anoxic pre-conditioning in diabetic cardiomyopathy. J Endocrinol Invest 31: 103-110.

20. Li Y, Lu Z, Keogh CL, Yu SP, Wei L (2007) Erythropoietin-induced neurovascular protection, angiogenesis, and cerebral blood flow restoration after focal ischemia in mice. J Cereb Blood Flow Metab 27: 1043-1054.

21. Haider KH, Ashraf M (2012) Preconditioning approach in stem cell therapy for the treatment of infarcted heart. Prog Mol Biol Transl 111: 323-356.

22. Yan F, Yao Y, Chen L, Li Y, Sheng Z, et al. (2012) Hypoxic preconditioning improves survival of cardiac progenitor cells: role of stromal cell derived factor-1a-CXCR4 axis. PloS one 7: e37948.

23. Zhang J, Chen GH, Wang YW, Zhao J, Duan HF, et al. (2012) Hydrogen peroxide preconditioning enhances the therapeutic efficacy of Wharton's Jelly mesenchymal stem cells after myocardial infarction. Chin Med J 125: 3472 3478 .

24. Murphy M, Hinman A, Levine AJ (1996) Wild-type p53 negatively regulates the expression of a microtubule-associated protein. Genes Dev 10: 2971 2980.

25. Vikhanskaya F, Vignati S, Beccaglia P, Ottoboni C, Russo P, et al. (1998) Inactivation of p53 in a human ovarian cancer cell line increases the sensitivity to paclitaxel by inducing G2/M arrest and apoptosis. Exp Cell Res 241: 96101.

26. Chen HM, Zhang DG, Wu JX, Pei DS, Zheng JN (2014) Ubiquitination of p53 is involved in troglitazone induced apoptosis in cervical cancer cells. Asian Pac J Cancer Prev 15: 2313-2318.

27. Zhou F, Yang Y, Xing D (2011) Bcl-2 and Bcl-xL play important roles in the crosstalk between autophagy and apoptosis. FEBS J 278: 403-413.

28. Shimizu S, Eguchi Y, Kamiike W, Itoh Y, Hasegawa J, et al. (1996) Induction of apoptosis as well as necrosis by hypoxia and predominant prevention of apoptosis by Bcl-2 and Bcl-XL. Cancer Res 56: 2161-2166.

29. Papandreou I, Krishna C, Kaper F, Cai D, Giaccia AJ, et al. (2005) Anoxia is necessary for tumor cell toxicity caused by a low-oxygen environment. Cancer Res 65: 3171-3178.

30. Weinmann M, Jendrossek V, Handrick R, Guner D, Goecke B, et al. (2004) Molecular ordering of hypoxia-induced apoptosis: critical involvement of the mitochondrial death pathway in a FADD/caspase-8 independent manner. Oncogene 23: 3757-3769.

31. Leonard MO, Cottell DC, Godson C, Brady HR, Taylor CT (2003) The role of HIF-1 alpha in transcriptional regulation of the proximal tubular epithelial cell response to hypoxia. J Biol Chem 278: 40296-40304.

32. Schmaltz C, Hardenbergh PH, Wells A, Fisher DE (1998) Regulation of proliferation-survival decisions during tumor cell hypoxia. Mol Cell Biol 18: 2845-2854.

33. Kim JY, Ahn HJ, Ryu JH, Suk K, Park JH (2004) BH3-only protein Noxa is a mediator of hypoxic cell death induced by hypoxia-inducible factor 1alpha. $J$ Exp Med 199: 113-124.

34. Buckley CD, Ross EA, McGettrick HM, Osborne CE, Haworth O, et al. (2006) Identification of a phenotypically and functionally distinct population of longlived neutrophils in a model of reverse endothelial migration. J Leukoc Biol 79: 303-311.

35. Buttke TM, Sandstrom PA (1994) Oxidative stress as a mediator of apoptosis. Immunol Today 15: 7-10

36. Dragon S, Saffar AS, Shan L, Gounni AS (2008) IL-17 attenuates the antiapoptotic effects of GM-CSF in human neutrophils. Mol Immunol 45: 160-168.

37. Arruda MA, Barcellos-de-Souza P, Sampaio AL, Rossi AG, Graca-Souza AV, et al. (2006) NADPH oxidase-derived ROS: key modulators of heme-induced mitochondrial stability in human neutrophils. Exp Cell Res 312: 3939-3948.

38. Dyugovskaya L, Polyakov A, Lavie P, Lavie L (2008) Delayed neutrophil apoptosis in patients with sleep apnea. Am J Respir Crit Care Med 177: $544-$ 554.

39. Chao DT, Korsmeyer SJ (1998) BCL-2 family: regulators of cell death. Annu Rev Immunol 16: 395-419. 
Citation: Han YS, Lee JH, Lee SH. Regulation of Hypoxia-Induced Cell Death and Application of Hypoxic Preconditioning to Stem Cell Transplantation. J Transplant Stem Cel Biol. 2014;2(1): 5

ISSN: 2374-9326

40. Fossati G, Moulding DA, Spiller DG, Moots RJ, White MR, et al. (2003) The mitochondrial network of human neutrophils: role in chemotaxis, phagocytosis, respiratory burst activation, and commitment to apoptosis. J Immunol 170: 1964-1972.

41. Frasch SC, Nick JA, Fadok VA, Bratton DL, Worthen GS, et al. (1998) p38 mitogen-activated protein kinase-dependent and -independent intracellula signal transduction pathways leading to apoptosis in human neutrophils. $J$ Biol Chem 273: 8389-8397.

42. Fudala R, Krupa A, Matthay MA, Allen TC, Kurdowska AK (2007) Anti-IL-8 autoantibody:IL-8 immune complexes suppress spontaneous apoptosis of neutrophils. Am J Physiol Lung Cell Mol Physiol 293: L364-L374.

43. Genestier AL, Michallet MC, Prevost G, Bellot G, Chalabreysse L, et al (2005) Staphylococcus aureus Panton-Valentine leukocidin directly targets mitochondria and induces Bax-independent apoptosis of human neutrophils. J Clin Invest 115: 3117-3127.

44. Gozal E, Sachleben LR Jr, Rane MJ, Vega C, Gozal D (2005) Mild sustained and intermittent hypoxia induce apoptosis in PC-12 cells via different mechanisms. Am J Physiol Cell Physiol 288: C535- C542.

45. Htoo AK, Greenberg H, Tongia S, Chen G, Henderson T, et al. (2006) Activation of nuclear factor kappaB in obstructive sleep apnea: a pathway leading to systemic inflammation. Sleep Breath 10: 43-50.

46. Karlsson A, Dahlgren C (2002) Assembly and activation of the neutrophil NADPH oxidase in granule membranes. Antioxid Redox Signal 4: 49-60.

47. Kasahara Y, Iwai K, Yachie A, Ohta K, Konno A, et al. (1997) Involvement of reactive oxygen intermediates in spontaneous and CD95 (Fas/APO-1)mediated apoptosis of neutrophils. Blood 89: 1748-1753.

48. Kato T, Kutsuna H, Oshitani N, Kitagawa S (2006) Cyclic AMP delays neutrophil apoptosis via stabilization of Mcl-1. FEBS Lett 580: 4582-4586.

49. Lenihan CR, Taylor CT (2013) The impact of hypoxia on cell death pathways. Biochem Soc Trans 41: 657-663.

50. Aylon Y, Oren M (2007) Living with p53, dying of p53. Cell 130: 597-600

51. Koumenis C, Alarcon R, Hammond E, Sutphin P, Hoffman W, et al. (2001) Regulation of p53 by hypoxia: dissociation of transcriptional repression and apoptosis from p53-dependent transactivation. Mol Cell Biol 21: 1297-1310.

52. Haider H, Ashraf M (2008) Strategies to promote donor cell survival: combining preconditioning approach with stem cell transplantation. J Mol Cell Cardiol 45: 554-566.

53. Afzal MR, Haider H, Idris NM, Jiang S, Ahmed RP, et al. (2010) Preconditioning promotes survival and angiomyogenic potential of mesenchymal stem cells in the infarcted heart via NF-kappaB signaling. Antioxid Redox Signal 12: 693-

54. Liu H, Xue W, Ge G, Luo X, Li Y, et al. (2010) Hypoxic preconditioning advances CXCR4 and CXCR7 expression by activating HIF-1alpha in MSCs. Biochem Biophys Res Commun 401: 509-515.

55. Doorn J, Moll G, Le Blanc K, van Blitterswijk C, de Boer J (2012) Therapeutic applications of mesenchymal stromal cells: paracrine effects and potentia improvements. Tissue Eng Part B Rev 18: 101-115.

56. Yao Y, Zhang F, Wang L, Zhang G, Wang Z, et al. (2009) Lipopolysaccharide preconditioning enhances the efficacy of mesenchymal stem cells transplantation in a rat model of acute myocardial infarction. J Biomed Sci 16: 74.

57. Zeng X, Yu SP, Taylor T, Ogle M, Wei L (2012) Protective effect of apelin on cultured rat bone marrow mesenchymal stem cells against apoptosis. Stem Cell Res 8: 357-367.

58. Wisel S, Khan M, Kuppusamy ML, Mohan IK, Chacko SM, et al. (2009) Pharmacological preconditioning of mesenchymal stem cells with trimetazidine (1-[2,3,4-trimethoxybenzyl]piperazine) protects hypoxic cells against oxidative stress and enhances recovery of myocardial function in infarcted heart through Bcl-2 expression. J Pharmacol Exp Ther 329: 543550

59. Choi KE, Hall CL, Sun JM, Wei L, Mohamad O, et al. (2012) A novel stroke therapy of pharmacologically induced hypothermia after focal cerebral ischemia in mice. FASEB J 26: 2799-2810.

60. Sims B, Clarke M, Francillion L, Kindred E, Hopkins ES, et al. (2012) Hypoxic preconditioning involves system Xc- regulation in mouse neural stem cells. Stem Cell Res 8: 285-291.

61. Shih AY, Erb H, Sun X, Toda S, Kalivas PW, et al. (2006) Cystine/glutamate exchange modulates glutathione supply for neuroprotection from oxidative stress and cell proliferation. J Neurosci 26: 10514-10523.

62. Cerrada I, Ruiz-Sauri A, Carrero R, Trigueros C, Dorronsoro A, et al (2013) Hypoxia-inducible factor 1 alpha contributes to cardiac healing in mesenchymal stem cells-mediated cardiac repair. Stem Cells Dev 22: 501511.

63. Wei L, Fraser JL, Lu ZY, Hu X, Yu SP (2012) Transplantation of hypoxia preconditioned bone marrow mesenchymal stem cells enhances angiogenesis and neurogenesis after cerebral ischemia in rats. Neurobiol Dis 46: $635-645$.

64. Theus MH, Wei L, Cui L, Francis K, Hu X, et al. (2008) In vitro hypoxic preconditioning of embryonic stem cells as a strategy of promoting cell survival and functional benefits after transplantation into the ischemic rat brain. Exp Neurol 210: 656-670.

65. Francis KR, Wei L (2010) Human embryonic stem cell neural differentiation and enhanced cell survival promoted by hypoxic preconditioning. Cell Death Dis 1: e22.

66. Cui L, Jiang J, Wei L, Zhou X, Fraser JL, et al. (2008) Transplantation of embryonic stem cells improves nerve repair and functional recovery after severe sciatic nerve axotomy in rats. Stem cells 26: 1356-1365.

67. Ogle ME, Yu SP, Wei L (2009) Primed for lethal battle: a step forward to enhance the efficacy and efficiency of stem cell transplantation therapy. J Thorac Cardiovasc Surg 138: 527.

68. Ong LL, Li W, Oldigs JK, Kaminski A, Gerstmayer B, et al. (2010) Hypoxic/ normoxic preconditioning increases endothelial differentiation potential of human bone marrow CD133+ cells. Tissue Eng Part C Methods 16: 10691081.

69. Akita T, Murohara T, Ikeda H, Sasaki K, Shimada T, et al. (2003) Hypoxic preconditioning augments efficacy of human endothelial progenitor cells for therapeutic neovascularization. Lab Invest 83: 65-73.

70. Li WL, Fraser JL, Yu SP, Zhu J, Jiang YJ, et al. (2011) The role of VEGF/ VEGFR2 signaling in peripheral stimulation-induced cerebral neurovascular regeneration after ischemic stroke in mice. Exp Brain Res 214: 503-513.

71. Leroux L, Descamps B, Tojais NF, Seguy B, Oses P, et al. (2010) Hypoxia preconditioned mesenchymal stem cells improve vascular and skeletal muscle fiber regeneration after ischemia through a Wnt4-dependent pathway. Mol Ther 18: 1545-1552.

72. Yamazaki M, Nakamura K, Mizukami Y, li M, Sasajima J, et al. (2008) Sonic hedgehog derived from human pancreatic cancer cells augments angiogenic function of endothelial progenitor cells. Cancer Sci 99: 1131-1138.

73. Volkmer E, Kallukalam BC, Maertz J, Otto S, Drosse I, et al. (2010) Hypoxic preconditioning of human mesenchymal stem cells overcomes hypoxiainduced inhibition of osteogenic differentiation. Tissue Eng Part A 16: 153164.

\section{Acknowledgements}

This study was supported by a National Research Foundation (NRF) grant funded by the Korean government (MEST) (2011-0009610). The funders had no role in study design, data collection or analysis, the decision to publish, or preparation of the manuscript. 